\title{
An Expeditious and Safe Synthesis of Some Exocyclic $\alpha, \beta$-Unsaturated Ketones by Microwave-Assisted Condensation of Cyclic Ketones with Aromatic Aldehydes over Anhydrous Potassium Carbonate
}

\author{
Rina Mondal, Tapas K. Mandal, and Asok K. Mallik \\ Department of Chemistry, Jadavpur University, Kolkata 700 032, India \\ Correspondence should be addressed to Asok K. Mallik, mallikak52@yahoo.co.in \\ Received 4 October 2012; Revised 29 November 2012; Accepted 2 December 2012 \\ Academic Editor: Vito Ferro \\ Copyright ( $) 2012$ Rina Mondal et al. This is an open access article distributed under the Creative Commons Attribution License, \\ which permits unrestricted use, distribution, and reproduction in any medium, provided the original work is properly cited.

\begin{abstract}
A rapid, efficient, and solvent-free methodology for synthesis of exocyclic $\alpha, \beta$-unsaturated ketones of the categories $E$-3arylidene-4-chromanones, $E$-2-arylidene-1-tetralones, $E$-2-arylidene-1-indanones, $E$-3-cinnamylidene-4-chromanones, $E$-2cinnamylidene-1-tetralones, $\quad E$-2-cinnamylidene-1-indanones, $\alpha, \alpha^{\prime}-(E, E)$-bis(arylidene)-cycloalkanones, and $\alpha, \alpha^{\prime}$-(E,E)-bis (cinnamylidene)-cycloalkanones has been developed through cross-aldol condensation of the constituent cyclic ketones and aldehydes by microwave irradiation over anhydrous potassium carbonate. However, for condensation of 1-thio-4-chromanones with aromatic aldehydes by this method, the initially formed exocyclic $\alpha, \beta$-unsaturated ketone has been found to undergo isomerization yielding 3-(arylmethyl)thiochromones.
\end{abstract}

\section{Introduction}

Exocyclic $\alpha, \beta$-unsaturated ketones are very suitable starting materials for synthesis of versatile heterocycles having polycyclic skeletons. Their cyclocondensation with dinucleophiles constitutes an important route to polycyclic fused ring systems, for example, tricyclic pyrazolines, tetracyclic benzothiazepines, tetracyclic benzodiazepines, thiazines, pyrimidines, quinazolines, and so forth [1-4]. Again, their 1,3-dipolar cycloaddition with different dipoles provides important nitrogen-containing spiroheterocycles [2]. Moreover, there is scope for performing a Michael addition reaction at the active double bond present in them. Some of the exocyclic $\alpha, \beta$-unsaturated ketones, namely, aurones and E-3-benzylidenechromanones, are natural compounds $[1,5-7]$. It may be mentioned here that several classes of compounds belonging to this category show interesting biological properties, for example, $\alpha, \alpha^{\prime}-(E, E)$-bis(arylidene)cycloalkanones show antiangiogenic $[8,9]$, quinine reductase inducer [10], arginine methyltransferase inhibitor [11], cytotoxic [12, 13], cholesterol-lowering [14], and antitubercular [15] activities, and E-3-benzylidenechromanones and related homoisoflavonoids show antioxidant, antiinflammatory, antifungal, antiviral, antiallergic, antihistaminic, antirhinovirus, antimutagenic, angioproductive, hypocholesterolemic, and cytotoxic activities $[7,16]$. Moreover, $\alpha, \alpha^{\prime}-(E, E)$-bis(arylidene)-cycloalkanones find application as fluorescent materials [17] and in the preparation of nonlinear optical materials and liquid-crystalline polymers [18]. All these aspects have made exocyclic $\alpha, \beta$-unsaturated ketones important synthetic targets for organic chemists. The reported methods for their synthesis up to 2003 have been reviewed by Lévai [1]. There has been addition of a good number of other methods in the literature subsequently $[7,9,17-37]$. However, some of the methods available so far suffer from drawbacks like use of toxic or corrosive reagents, expensive catalysts, hazardous solvents, long reaction times, tedious isolation procedure, and so forth, due to which the current literature shows a growing trend for developing environmentally benign methodologies for synthesis of 


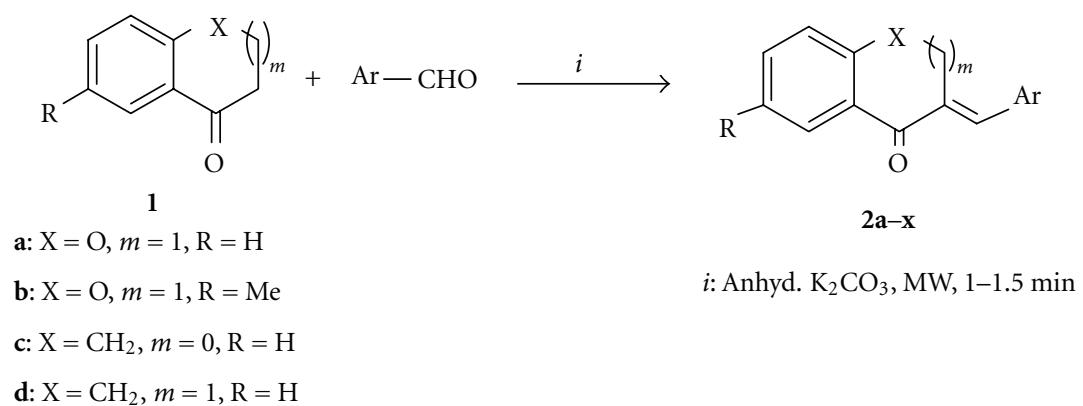<smiles>CC(C)C=O</smiles>

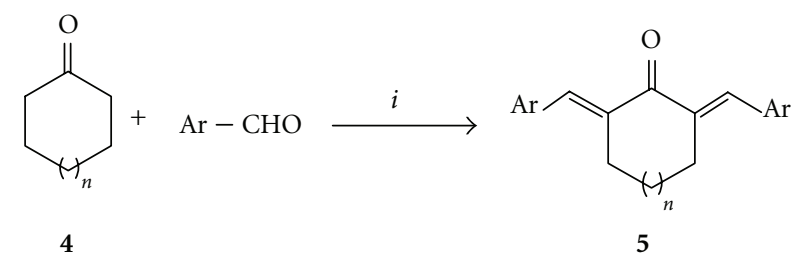

a: $n=0, \mathbf{b}: n=1, \mathbf{c}: n=2$

Scheme 1: Condensation of cyclic ketones with aromatic aldehydes.

different exocyclic $\alpha, \beta$-unsaturated ketones [7, 18, 23, 33$35,37]$. Mention of few such recent methods for synthesis of chalcones [38-43], a group of structurally related acyclic compounds, may be done in this connection. Microwave (MW) irradiation, an unconventional energy source, has been used for a variety of applications including organic synthesis, wherein chemical reactions are accelerated because of selective absorption of MW energy by polar molecules. This technology has opened up new opportunities to the synthetic chemists since mid-1980s, in the form of new reactions that are not possible by use of conventional heating. Its important advantages are being improved reaction yields, decreased reaction times, and safe performance of some reactions even under solvent-free reaction conditions. All these advantageous features have resulted in publication of a huge number of original research papers and a good number of reviews and monographs in the area during the last twenty five years [44-57]. A number of environmentally benign methodologies for condensation reactions leading to exocyclic $\alpha, \beta$-unsaturated ketones and chalcones are reported to be assisted by microwave [39-43]. Very recently, we have developed a method for synthesis of flavanones directly from $2^{\prime}$-hydroxyacetophenones and benzaldehydes by potassium carbonate catalyzed microwave-assisted condensation [58], the first step of which involves the occurrence of a Claisen-Schmidt reaction. Moreover, the appearance of several papers on utilization of anhydrous potassium carbonate for synthesis of $\alpha, \beta$-unsaturated ketones is evident from the recent literature $[23,41,42]$. All these aspects interested us to apply the very simple methodology developed by us [58] for the synthesis of exocyclic $\alpha, \beta$-unsaturated ketones. Thus, we took cyclic methyleneketones belonging to the categories 4-chromanone, 1-thio-4-chromanone, 1-tetralone, 1 -indanone, and cycloalkanones as starting materials for getting the corresponding exocyclic $\alpha, \beta$-unsaturated ketones (Scheme 1). The results of this study have been presented herein.

\section{Results and Discussion}

By following our recent methodology [58], when an equimolar mixture of an aromatic aldehyde and chromanone (1a or 1b)/1-tetralone (1c)/1-indanone (1d)/1-thio-4-chromanone (1e) was subjected to microwave irradiation over anhydrous potassium carbonate, reaction took place completely within $1-1.5$ min yielding only one product in each case. For combinations where liquid aldehydes were used, a mixture of neutral alumina and anhydrous potassium carbonate was taken instead of anhydrous potassium carbonate alone. Some representative examples of microwave irradiation over neutral alumina alone done by us were found to give the condensation products in much lower yields (42\%-55\%). This observation has analogy with that reported in a recent paper by Kakati and Sarma [43] for synthesis of chalcones. Isolation of the product done by washing the solid obtained after the MW irradiation with dichloromethane followed by chromatography of the concentrate of the washings gave 
the desired condensation products from 1a-d (Table 1). When le was used as substrate and condensation reaction was studied with benzaldehyde, 4-chlorobenzaldehyde, and 4-methoxybenzaldehyde (irradiation time $1.5 \mathrm{~min}$ ), it was found that the initially formed exocyclic $\alpha, \beta$-unsaturated ketone underwent complete isomerization in the first two cases yielding corresponding 3-benzylthiochromones (3a and $3 \mathbf{b}$ ), while the desired product $2 \mathbf{y}$ was obtained in the last case (Table 2). The role of the electron donating $p$-OMe group in $2 y$ in inhibiting the double bond isomerization was thus evident. The same reaction done on neutral alumina, however, gave the desired exocyclic $\alpha, \beta$-unsaturated ketones $(2 \mathbf{y}-\mathbf{z 1})$, albeit only in moderate yield (Table 2$)$. Attempted synthesis of $E$-3-benzylideneflavanones by condensation of flavanone and benzaldehydes by the use of this methodology, however, did not meet with success.

Considering the importance of $\alpha, \alpha^{\prime}$-(E,E)-bis (arylidene)-cycloalkanones (5) as mentioned in the introduction, our study was then directed to the reactions involving cycloalkanones (4) as ketomethylene component. Thus, condensation of each of cyclopentanone (4a), cyclohexanone (4b), and cycloheptanone (4c) with 2 molar proportion of simple aromatic aldehydes was also found to produce exocyclic $\alpha, \beta$-unsaturated ketones $\mathbf{5 a - j}$ in very good yield (Table 3). Reactions of these cycloalkanones with 1 molar proportion of aromatic aldehyde under the previously said irradiation condition were found to produce $\alpha, \alpha^{\prime}-(E, E)$ bis(arylidene)-cycloalkanones (yield: $38 \%-45 \%$ ) instead of any monoarylidene product. Our attempts to apply this methodology for condensation of each of the ketones 1a and 4 with the aliphatic aldehydes heptanal and citral, however, did not meet with success.

\section{Conclusion}

Microwave irradiation of cyclic ketones and aromatic aldehydes over anhydrous potassium carbonate has been developed as a new methodology for the synthesis of several series of exocyclic $\alpha, \beta$-unsaturated ketones. The method is very efficient, simple, and environmentally benign.

\section{Experimental}

4.1. General. Melting points were recorded on a Köfler block. IR spectra were recorded on a Perkin Elmer FT-IR spectrophotometer (Spectrum BX II) in $\mathrm{KBr}$ pellets. ${ }^{1} \mathrm{H}$ and ${ }^{13} \mathrm{C}$ NMR spectra were recorded in $\mathrm{CDCl}_{3}$ on a Bruker AV-300 $(300 \mathrm{MHz})$ spectrometer. Analytical samples were routinely dried in vacuo at room temperature. Microanalytical data were recorded on a Perkin-Elmer 2400 Series II C, H, N analyzer. Mass spectra were measured in the following ways: ESIMS(+) [Waters Micromass Q-Tof micro] and FAB-MS [Jeol the M Station JMS.700]. An unmodified domestic household microwave oven (LG, DMO, Model No.-556P, 900 watt) equipped with inverter technology, which provides a realistic control of the microwave power to the desired level $(20 \%-100 \%)$, was used for microwave heating. The MW oven was operated at reduced MW-power level of 60\% (540 watt). Column chromatography was performed with silica gel (100-200 mesh), and TLC with silica gel G made of SRL Pvt. Ltd. Petroleum ether had the boiling range $60-80^{\circ} \mathrm{C}$.

4.2. General Procedure for Condensation of Ketones with Aldehydes. A solution of a mixture of ketone (1) (1 mmol) and aldehyde $(1 \mathrm{mmol} / 2 \mathrm{mmol})$ in $\mathrm{CH}_{2} \mathrm{Cl}_{2}$ was added to a mixture of anhyd. $\mathrm{K}_{2} \mathrm{CO}_{3}(1 \mathrm{~g})$ (neutral alumina (1.5 g) was added when liquid aldehyde was used), and the solvent was removed. The solid material thus obtained was subjected to microwave irradiation $(2450 \mathrm{MHz})$ at temperatures $92^{\circ} \mathrm{C}$ (for $1.5 \mathrm{~min}$ ) and $82^{\circ} \mathrm{C}$ (for $1 \mathrm{~min}$ ), monitoring the progress of reaction by TLC. It was then washed thoroughly with $\mathrm{CH}_{2} \mathrm{Cl}_{2}$, and the concentrate of the washings was chromatographed over silica gel to obtain pure product. All the exocyclic $\alpha, \beta$-unsaturated ketones (2 (Tables 1 and 2) and 5 (Table 3), all light yellow crystals) as well as the 3benzylthiochromones (3 (Table 2), very light yellow crystals) obtained were properly characterized from their physical, analytical, and spectral data (majority of these compounds were known previously). The analytical and spectral data of some selected compounds are given in the following.

4.2.1. Compound 2i. IR $\left(\mathrm{KBr}, \mathrm{cm}^{-1}\right)$ : $1669(\mathrm{C}=\mathrm{O}) ;{ }^{1} \mathrm{H}$ NMR $\left(300 \mathrm{MHz}, \mathrm{CDCl}_{3}, \delta / \mathrm{ppm}\right): 2.34(\mathrm{~s}, 3 \mathrm{H}, \mathrm{Me}), 5.28\left(\mathrm{~s}, 2 \mathrm{H}, \mathrm{H}_{2}-\right.$ 2), $6.87(\mathrm{~d}, 1 \mathrm{H}, J=8.4 \mathrm{~Hz}, \mathrm{H}-8), 7.19-7.32(\mathrm{~m}, 3 \mathrm{H}, \mathrm{H}-7, \mathrm{H}-$ $2^{\prime}$ and $\left.\mathrm{H}-6^{\prime}\right), 7.43\left(\mathrm{~d}, 2 \mathrm{H}, J=8.4 \mathrm{~Hz}, \mathrm{H}-3^{\prime}\right.$ and $\left.\mathrm{H}-5^{\prime}\right), 7.80$ (br. s, $2 \mathrm{H}, \mathrm{H}-\beta$ and $\mathrm{H}-5$ ).

4.2.2. Compound 2l. IR $\left(\mathrm{KBr}, \mathrm{cm}^{-1}\right): 1670(\mathrm{C}=\mathrm{O}) ;{ }^{1} \mathrm{H}$ NMR $\left(300 \mathrm{MHz}, \mathrm{CDCl}_{3}, \delta / \mathrm{ppm}\right): 2.33$ (s, $\left.3 \mathrm{H}, \mathrm{Me}\right), 5.31\left(\mathrm{~s}, 2 \mathrm{H}, \mathrm{H}_{2}-\right.$ 2), $6.03\left(\mathrm{~s}, 2 \mathrm{H},-\mathrm{OCH}_{2} \mathrm{O}-\right), 6.79-6.90(\mathrm{~m}, 4 \mathrm{H}, \mathrm{Ar}-\mathrm{H}), 7.25-$ $7.30(\mathrm{~m}, 1 \mathrm{H}, \mathrm{H}-7), 7.77$ (br. s, $1 \mathrm{H}, \mathrm{H}-\beta / \mathrm{H}-5), 7.80$ (br. s, $1 \mathrm{H}$, $\mathrm{H}-5 / \mathrm{H}-\beta)$.

4.2.3. Compound 2n. IR $\left(\mathrm{KBr}, \mathrm{cm}^{-1}\right): 1662(\mathrm{C}=\mathrm{O}) ;{ }^{1} \mathrm{H}$ NMR $\left(300 \mathrm{MHz}, \mathrm{CDCl}_{3}, \delta / \mathrm{ppm}\right): 2.34(\mathrm{~s}, 3 \mathrm{H}, \mathrm{Me}), 3.86(\mathrm{~s}, 3 \mathrm{H}$, $\mathrm{OMe}$ ), 5.34 (br. s, $\left.2 \mathrm{H}, \mathrm{H}_{2}-2\right), 6.87(\mathrm{~d}, 1 \mathrm{H}, J=8.9 \mathrm{~Hz}, \mathrm{H}-8)$, $6.97\left(\mathrm{~d}, 2 \mathrm{H}, J=8.9 \mathrm{~Hz}, \mathrm{H}-3^{\prime}, 5^{\prime}\right), 7.27-7.31(\mathrm{~m}, 3 \mathrm{H}, \mathrm{H}-7$ and H-2', $6^{\prime}$ ), 7.80 (br. s, $1 \mathrm{H}, \mathrm{H}-\beta / \mathrm{H}-5$ ), 7. 83 (br. s, $1 \mathrm{H}, \mathrm{H}-5 / \mathrm{H}-$ $\beta)$.

4.2.4. Compound 2r. IR $\left(\mathrm{KBr}, \mathrm{cm}^{-1}\right): 1669 \mathrm{~cm}^{-1}(\mathrm{C}=\mathrm{O}) ;{ }^{1} \mathrm{H}$ $\operatorname{NMR}\left(300 \mathrm{MHz}, \mathrm{CDCl}_{3}, \delta / \mathrm{ppm}\right): 2.96\left(\mathrm{t}, 2 \mathrm{H}, J=6.9 \mathrm{~Hz}, \mathrm{H}_{2}-\right.$ $\left.3 / \mathrm{H}_{2}-4\right), 3.17\left(\mathrm{t}, 2 \mathrm{H}, J=6.0 \mathrm{~Hz}, \mathrm{H}_{2}-4 / \mathrm{H}_{2}-3\right), 3.87(\mathrm{~s}, 3 \mathrm{H}$, $\mathrm{OMe}), 6.97\left(\mathrm{~d}, 2 \mathrm{H}, J=8.7 \mathrm{~Hz}, \mathrm{H}-3^{\prime}, 5^{\prime}\right), 7.27(\mathrm{~d}, 1 \mathrm{H}, J=$ $7.8 \mathrm{~Hz}, \mathrm{H}-5), 7.38(\mathrm{t}, 1 \mathrm{H}, J=7.5 \mathrm{~Hz}, \mathrm{H}-7), 7.45(\mathrm{~d}, 2 \mathrm{H}, J=$ $\left.8.7 \mathrm{~Hz}, \mathrm{H}-2^{\prime}, 6^{\prime}\right), 7.50(\mathrm{dt}, 1 \mathrm{H}, J=7.5$ and $1.0 \mathrm{~Hz}, \mathrm{H}-6), 7.87$ (br. s, $1 \mathrm{H}, \mathrm{H}-\beta$ ), 8.14 (br. d, $1 \mathrm{H}, J=7.8 \mathrm{~Hz}, \mathrm{H}-8$ ).

4.2.5. Compound 2s. IR $\left(\mathrm{KBr}, \mathrm{cm}^{-1}\right): 1666 \mathrm{~cm}^{-1}(\mathrm{C}=\mathrm{O}) ;{ }^{1} \mathrm{H}$ $\operatorname{NMR}\left(300 \mathrm{MHz}, \mathrm{CDCl}_{3}, \delta / \mathrm{ppm}\right): 2.96\left(\mathrm{t}, 2 \mathrm{H}, J=6.3 \mathrm{~Hz}, \mathrm{H}_{2}-\right.$ $3 / \mathrm{H}_{2}-4$ ), 3.09 (t, $2 \mathrm{H}, J=6.1 \mathrm{~Hz}, \mathrm{H}_{2}-4 / \mathrm{H}_{2}-3$ ), 7.25 (br. d, $1 \mathrm{H}$, $J=7.5 \mathrm{~Hz}$ ), 7.30 (br. d, $2 \mathrm{H}, J=8.1 \mathrm{~Hz}, \mathrm{H}-3^{\prime}, 5^{\prime}$ ), 7.37 (br. t, $1 \mathrm{H}, J=7.6 \mathrm{~Hz}$ ), 7.50 (br. t, $1 \mathrm{H}, J=7.4 \mathrm{~Hz}, \mathrm{H}-6), 7.55$ (d, $2 \mathrm{H}$, $J=8.4 \mathrm{~Hz}, \mathrm{H}-2^{\prime}, 6^{\prime}$ ), 7.77 (br. s, $1 \mathrm{H}, \mathrm{H}-\beta$ ), 8.13 (br. d, $1 \mathrm{H}, J=$ $7.7 \mathrm{~Hz}, \mathrm{H}-8$ ). 
TABLE 1: Microwave-assisted condensation of chromanones/1-tetralone/1-indanone with aromatic aldehydes.

\begin{tabular}{|c|c|c|c|c|c|c|}
\hline Entry & $\begin{array}{l}\text { Ketone } \\
(1)\end{array}$ & Ar of aldehyde & $\begin{array}{l}\text { Time } \\
(\mathrm{min})\end{array}$ & $\begin{array}{l}\text { Product } \\
\quad(2)\end{array}$ & Yield (\%) & $\begin{array}{c}\text { M.P. }\left({ }^{\circ} \mathrm{C}\right) \\
\text { Obs. (Lit.) }[\text { Reference }]\end{array}$ \\
\hline (1) & 1a & $\mathrm{C}_{6} \mathrm{H}_{5}-$ & 1.5 & $2 a$ & $\begin{array}{l}82^{\mathrm{a}} \\
45^{\mathrm{b}}\end{array}$ & $\begin{array}{c}111 \\
(110)[59]\end{array}$ \\
\hline (2) & 1a & $4-\mathrm{Cl}-\mathrm{C}_{6} \mathrm{H}_{4}-$ & 1.5 & $2 b$ & $\begin{array}{l}88^{\mathrm{a}} \\
49^{\mathrm{b}}\end{array}$ & $\begin{array}{l}169-170 \\
(168)[59]\end{array}$ \\
\hline (3) & 1a & $4-\mathrm{MeO}-\mathrm{C}_{6} \mathrm{H}_{4}-$ & 1.0 & $2 c$ & $\begin{array}{l}79^{\mathrm{a}} \\
43^{\mathrm{b}}\end{array}$ & $\begin{array}{c}131 \\
\text { (132) [59] }\end{array}$ \\
\hline (4) & 1a & $4-\mathrm{Br}-\mathrm{C}_{6} \mathrm{H}_{4}-$ & 1.0 & $2 d$ & $\begin{array}{l}86^{\mathrm{a}} \\
55^{\mathrm{b}}\end{array}$ & $\begin{array}{c}175 \\
(174)[59]\end{array}$ \\
\hline (5) & 1a & $4-\mathrm{Me}-\mathrm{C}_{6} \mathrm{H}_{4}-$ & 1.0 & $2 \mathrm{e}$ & $\begin{array}{l}77^{\mathrm{a}} \\
51^{\mathrm{b}}\end{array}$ & $\begin{array}{c}117 \\
(118)[59]\end{array}$ \\
\hline (6) & 1a & & 1.5 & $2 f$ & $74^{\mathrm{a}}$ & $\begin{array}{c}115 \\
(115)[59]\end{array}$ \\
\hline (7) & 1a & $E-\mathrm{C}_{6} \mathrm{H}_{5} \mathrm{CH}=\mathrm{CH}-$ & 1.5 & $2 \mathrm{~g}$ & $\begin{array}{l}87^{\mathrm{a}} \\
42^{\mathrm{b}}\end{array}$ & $\begin{array}{c}135-136 \\
(136)[59]\end{array}$ \\
\hline (8) & $1 b$ & $\mathrm{C}_{6} \mathrm{H}_{5}-$ & 1.0 & $2 \mathrm{~h}$ & $84^{\mathrm{a}}$ & $\begin{array}{c}132 \\
(133)[59]\end{array}$ \\
\hline (9) & $1 b$ & $4-\mathrm{Cl}-\mathrm{C}_{6} \mathrm{H}_{4}-$ & 1.5 & $2 \mathbf{i}$ & $89^{\mathrm{a}}$ & $\begin{array}{c}143-144 \\
(144)[59]\end{array}$ \\
\hline (10) & $1 b$ & $4-\mathrm{Me}-\mathrm{C}_{6} \mathrm{H}_{4}-$ & 1.5 & $2 \mathbf{j}$ & $76^{\mathrm{a}}$ & $\begin{array}{c}120 \\
(120)[59]\end{array}$ \\
\hline (11) & $1 b$ & $4-\mathrm{Me}_{2} \mathrm{~N}-\mathrm{C}_{6} \mathrm{H}_{4}-$ & 1.0 & $2 \mathrm{k}$ & $81^{\mathrm{a}}$ & $\begin{array}{c}140 \\
(140)[59]\end{array}$ \\
\hline (12) & $1 b$ & & 1.5 & 21 & $73^{\mathrm{a}}$ & $\begin{array}{c}134-135 \\
(135)[59]\end{array}$ \\
\hline (13) & $1 b$ & 2-Furanyl & 1.5 & $2 \mathrm{~m}$ & $80^{\mathrm{a}}$ & $\begin{array}{c}101 \\
(101)[42]\end{array}$ \\
\hline (14) & $1 b$ & $4-\mathrm{MeO}-\mathrm{C}_{6} \mathrm{H}_{4}-$ & 1.5 & $2 n$ & $72^{\mathrm{a}}$ & 104 \\
\hline (15) & $1 b$ & $E-\mathrm{C}_{6} \mathrm{H}_{5} \mathrm{CH}=\mathrm{CH}-$ & 1.5 & 20 & $85^{\mathrm{a}}$ & $\begin{array}{c}148 \\
(148)[59]\end{array}$ \\
\hline (16) & 1c & $\mathrm{C}_{6} \mathrm{H}_{5-}$ & 1.5 & $2 p$ & $74^{\mathrm{a}}$ & $\begin{array}{c}120 \\
(120-121)[60]\end{array}$ \\
\hline (17) & $1 c$ & $4-\mathrm{Cl}-\mathrm{C}_{6} \mathrm{H}_{4}-$ & 1.5 & $2 q$ & $\begin{array}{l}84^{\mathrm{a}} \\
51^{\mathrm{b}}\end{array}$ & $\begin{array}{c}133-134 \\
(132-133)[60]\end{array}$ \\
\hline (18) & $1 c$ & $4-\mathrm{MeO}-\mathrm{C}_{6} \mathrm{H}_{4}-$ & 1.5 & $2 \mathbf{r}$ & $\begin{array}{l}75^{\mathrm{a}} \\
46^{\mathrm{b}}\end{array}$ & $\begin{array}{c}88 \\
(87-88)[60]\end{array}$ \\
\hline (19) & 1c & $4-\mathrm{Br}-\mathrm{C}_{6} \mathrm{H}_{4}-$ & 1.5 & $2 s$ & $82^{\mathrm{a}}$ & 158 \\
\hline (20) & $1 \mathrm{c}$ & $E-\mathrm{C}_{6} \mathrm{H}_{5} \mathrm{CH}=\mathrm{CH}-$ & 1.5 & $2 t$ & $\begin{array}{l}82^{\mathrm{a}} \\
48^{\mathrm{b}}\end{array}$ & $\begin{array}{c}134 \\
(133-134)[61]\end{array}$ \\
\hline (21) & 1d & $4-\mathrm{Me}-\mathrm{C}_{6} \mathrm{H}_{4}-$ & 1.5 & $2 u$ & $76^{a}$ & $\begin{array}{c}139-140 \\
(137-138)[62]\end{array}$ \\
\hline (22) & $1 d$ & $4-\mathrm{MeO}-\mathrm{C}_{6} \mathrm{H}_{4}-$ & 1.5 & $2 v$ & $71^{a}$ & $\begin{array}{c}139-140 \\
(138-139)[62]\end{array}$ \\
\hline (23) & $1 d$ & $4-\mathrm{Br}-\mathrm{C}_{6} \mathrm{H}_{4}-$ & 1.5 & $2 w$ & $\begin{array}{l}83^{\mathrm{a}} \\
53^{\mathrm{b}}\end{array}$ & $188-189$ \\
\hline (24) & 1d & $E-\mathrm{C}_{6} \mathrm{H}_{5} \mathrm{CH}=\mathrm{CH}-$ & 1.5 & $2 x$ & $\begin{array}{l}88^{\mathrm{a}} \\
45^{\mathrm{b}}\end{array}$ & $\begin{array}{c}125 \\
(124-125)[61]\end{array}$ \\
\hline
\end{tabular}

${ }^{\mathrm{a}}$ Over anhy. $\mathrm{K}_{2} \mathrm{CO}_{3} ;{ }^{\mathrm{b}}$ over neutral alumina. 
TABLE 2: Microwave-assisted condensation of 1-thiochroman-4-one (1e) with aromatic aldehydes.

\begin{tabular}{|c|c|c|c|c|c|c|}
\hline Entry & Catalyst & Ar of aldehyde & $\begin{array}{l}\text { Time } \\
(\min .)\end{array}$ & Product & $\begin{array}{l}\text { Yield } \\
(\%)\end{array}$ & $\begin{array}{c}\text { M.P } \\
\text { Obs. (Lit.) [Reference] }\end{array}$ \\
\hline (1) & Anhy. $\mathrm{K}_{2} \mathrm{CO}_{3}$ & $\mathrm{C}_{6} \mathrm{H}_{5-}$ & 1.5 & $3 a$ & 72 & $\begin{array}{c}60 \\
(59-61)[63]\end{array}$ \\
\hline (2) & Anhy. $\mathrm{K}_{2} \mathrm{CO}_{3}$ & $4-\mathrm{Cl}-\mathrm{C}_{6} \mathrm{H}_{4}-$ & 1.5 & $3 b$ & 78 & 110 \\
\hline (3) & Anhy. $\mathrm{K}_{2} \mathrm{CO}_{3}$ & $4-\mathrm{MeO}-\mathrm{C}_{6} \mathrm{H}_{4}-$ & 1.5 & $2 y$ & 69 & $\begin{array}{l}112-113 \\
(113)[59]\end{array}$ \\
\hline (4) & Neutral alumina & $\mathrm{C}_{6} \mathrm{H}_{5-}$ & 1.5 & $2 z$ & 55 & $\begin{array}{l}104-105 \\
(105)[59]\end{array}$ \\
\hline (5) & Neutral alumina & $4-\mathrm{Cl}-\mathrm{C}_{6} \mathrm{H}_{4}-$ & 1.5 & $2 \mathrm{z} 1$ & 59 & $\begin{array}{c}138-139 \\
(138-139)[59]\end{array}$ \\
\hline (6) & Neutral alumina & $4-\mathrm{MeO}-\mathrm{C}_{6} \mathrm{H}_{4}-$ & 1.5 & $2 y$ & 52 & $\begin{array}{l}112-113 \\
(113)[59] \\
\end{array}$ \\
\hline
\end{tabular}

TABLE 3: Microwave-assisted condensation of cycloalkanones with aromatic aldehydes and cinnamaldehyde over anhy. $\mathrm{K}_{2} \mathrm{CO}_{3}$.

\begin{tabular}{|c|c|c|c|c|c|c|}
\hline Entry & $\begin{array}{c}\text { Ketone } \\
(1)\end{array}$ & Ar of aldehyde & $\begin{array}{l}\text { Time } \\
(\mathrm{min} .)\end{array}$ & $\begin{array}{l}\text { Product } \\
\text { (5) }\end{array}$ & $\begin{array}{c}\text { Yield } \\
(\%)\end{array}$ & $\begin{array}{c}\text { M.P } \\
\text { Obs. (Lit.) [Reference] }\end{array}$ \\
\hline (1) & $4 a$ & $\mathrm{C}_{6} \mathrm{H}_{5-}$ & 1.5 & $5 a$ & 85 & $\begin{array}{c}190 \\
(190)[64]\end{array}$ \\
\hline (2) & $4 a$ & $4-\mathrm{MeO}-\mathrm{C}_{6} \mathrm{H}_{4}-$ & 1.5 & $5 b$ & 78 & $\begin{array}{c}215 \\
(215)[64]\end{array}$ \\
\hline (3) & $4 a$ & $4-\mathrm{Cl}-\mathrm{C}_{6} \mathrm{H}_{4}-$ & 1.5 & $5 c$ & 86 & $\begin{array}{c}228-229 \\
(229-230)[64]\end{array}$ \\
\hline (4) & $4 a$ & $E-\mathrm{C}_{6} \mathrm{H}_{5} \mathrm{CH}=\mathrm{CH}-$ & 1.5 & $5 d$ & 87 & $\begin{array}{c}220 \\
(220-221)[64]\end{array}$ \\
\hline (5) & $4 b$ & $\mathrm{C}_{6} \mathrm{H}_{5}-$ & 1.5 & $5 e$ & 82 & $\begin{array}{c}155 \\
(155)[64]\end{array}$ \\
\hline (6) & $4 b$ & $4-\mathrm{MeO}-\mathrm{C}_{6} \mathrm{H}_{4}-$ & 1.5 & $5 f$ & 78 & $\begin{array}{c}213 \\
(213)[64]\end{array}$ \\
\hline (7) & $4 \mathrm{~b}$ & $4-\mathrm{Cl}-\mathrm{C}_{6} \mathrm{H}_{4}-$ & 1.5 & $5 g$ & 88 & $\begin{array}{c}141-142 \\
(138)[64]\end{array}$ \\
\hline (8) & $4 b$ & $E-\mathrm{C}_{6} \mathrm{H}_{5} \mathrm{CH}=\mathrm{CH}-$ & 1.5 & $5 \mathrm{~h}$ & 82 & $\begin{array}{c}176-177 \\
(178)[64]\end{array}$ \\
\hline (9) & $4 c$ & $\mathrm{C}_{6} \mathrm{H}_{5-}$ & 1.5 & $5 i$ & 85 & $\begin{array}{c}108 \\
106.5-108[65]\end{array}$ \\
\hline (10) & $4 c$ & $E-\mathrm{C}_{6} \mathrm{H}_{5} \mathrm{CH}=\mathrm{CH}-$ & 1.5 & $5 \mathbf{j}$ & 81 & $\begin{array}{c}204-206 \\
207-208[65]\end{array}$ \\
\hline
\end{tabular}

4.2.6. Compound 2v. IR $\left(\mathrm{KBr}, \mathrm{cm}^{-1}\right): 1688 \mathrm{~cm}^{-1}(\mathrm{C}=\mathrm{O}) ;{ }^{1} \mathrm{H}$ $\mathrm{NMR}\left(300 \mathrm{MHz}, \mathrm{CDCl}_{3}, \delta / \mathrm{ppm}\right): 3.87$ (s, 3H, OMe), 4.02 (s, $\left.2 \mathrm{H}, \mathrm{H}_{2}-3\right), 6.98\left(\mathrm{~d}, 2 \mathrm{H}, J=8.7 \mathrm{~Hz}, \mathrm{H}-3^{\prime}, 5^{\prime}\right), 7.42$ (br. t, $1 \mathrm{H}$, $J=7.2 \mathrm{~Hz}, \mathrm{H}-6), 7.54-7.66(\mathrm{~m}, 5 \mathrm{H}, \mathrm{Ar}-\mathrm{H}$ and $\mathrm{H}-\beta), 7.91$ (br. d, $1 \mathrm{H}, J=7.5 \mathrm{~Hz}, \mathrm{H}-7) ;{ }^{13} \mathrm{C} \mathrm{NMR}(75 \mathrm{MHz}) \delta 32.47$, 55.40, 114.48, 124.30, 126.11, 127.58, 128.15, 132.41, 132.57, $133.81,134.34,138.25,149.50,160.87,194.39$.

4.2.7. Compound 2w. IR $\left(\mathrm{KBr}, \mathrm{cm}^{-1}\right): 1692 \mathrm{~cm}^{-1}(\mathrm{C}=\mathrm{O}) ;{ }^{1} \mathrm{H}$ $\mathrm{NMR}\left(300 \mathrm{MHz}, \mathrm{CDCl}_{3}, \delta / \mathrm{ppm}\right): 4.02$ (s, 2H, $\left.\mathrm{H}_{2}-2\right), 7.44$ (br. t, $1 \mathrm{H}, J=7.5 \mathrm{~Hz}, \mathrm{H}-6), 7.51-7.66$ (m, 7H, Ar-H and $\mathrm{H}-$ $\beta$ ), 7.91 (br. d, $1 \mathrm{H}, J=7.5 \mathrm{~Hz}, \mathrm{H}-7$ ).

4.2.8. Compound 2t. IR $\left(\mathrm{KBr}, \mathrm{cm}^{-1}\right): 1659 \mathrm{~cm}^{-1}(\mathrm{C}=\mathrm{O}) ;{ }^{1} \mathrm{H}$ $\mathrm{NMR}\left(300 \mathrm{MHz}, \mathrm{CDCl}_{3}, \delta / \mathrm{ppm}\right): 3.02$ (s, $4 \mathrm{H}, \mathrm{H}_{2}-3$ and $\mathrm{H}_{2}-$ 4), $7.04(\mathrm{~d}, 1 \mathrm{H}, J=15.3 \mathrm{~Hz}, \mathrm{H}-\delta), 7.17(\mathrm{dd}, 1 \mathrm{H}, J=15.3$ and
11.2 Hz, H- $\gamma$ ), 7.28-7.40 (m, 5H, Ar-H), 7.45-7.58 (m, 4H, $\mathrm{H}-6, \mathrm{H}-2^{\prime}, 6^{\prime}$ and $\left.\mathrm{H}-\beta\right), 8.11(\mathrm{dd}, 1 \mathrm{H}, J=7.5$ and $1.2 \mathrm{~Hz}$, $\mathrm{H}-8) ;{ }^{13} \mathrm{C} \mathrm{NMR}(75 \mathrm{MHz}) \delta: 25.97,28.72,123.40,126.95$, $127.14,128.07,128.15,128.77,128.86,133.00,133.78$, $134.39,135.89,136.62,140.93,143.37,187.27 . \mathrm{TOF} \mathrm{MS}^{+}: \mathrm{m} / z$ $283(\mathrm{M}+\mathrm{Na})^{+}$; Anal. Calcd for $\mathrm{C}_{19} \mathrm{H}_{16} \mathrm{O}$ (260.12): C, 87.66; H, 6.19\%. Found C, 87.48; H 6.34\%.

4.2.9. Compound $2 x$. IR $\left(\mathrm{KBr}, \mathrm{cm}^{-1}\right): 1693 \mathrm{~cm}^{-1}(\mathrm{C}=\mathrm{O}),{ }^{1} \mathrm{H}$ NMR (300 MHz, $\left.\mathrm{CDCl}_{3}, \delta / \mathrm{ppm}\right): 3.87$ (s, 2H, H2-3), 7.00$7.10(\mathrm{~m}, 2 \mathrm{H}, \mathrm{H}-\gamma$ and $\mathrm{H}-\delta), 7.32-7.44(\mathrm{~m}, 5 \mathrm{H}, \mathrm{Ar}-\mathrm{H}), 7.52-$ $7.63\left(\mathrm{~m}, 4 \mathrm{H}, \mathrm{H}-2^{\prime}, 6^{\prime}, \mathrm{H}-5\right.$ and $\left.\mathrm{H}-\beta\right), 7.87$ (br. d, $1 \mathrm{H}, J=$ $7.8 \mathrm{~Hz}, \mathrm{H}-7)$.

4.2.10. Compound 2y. IR $\left(\mathrm{KBr}, \mathrm{cm}^{-1}\right): 1661 \mathrm{~cm}^{-1}(\mathrm{C}=\mathrm{O})$; ${ }^{1} \mathrm{H}$ NMR $\left(300 \mathrm{MHz}, \mathrm{CDCl}_{3}, \delta / \mathrm{ppm}\right): 3.86$ (s, 3H), 4.16 
(s, 2H, H2-2), 6.96 (d, 2H, $\left.J=8.7 \mathrm{~Hz}, \mathrm{H}-3^{\prime}, 5^{\prime}\right), 7.23-7.41$ (m, 3H, H-6,7,8), 7.37 (d, 2H, J = 8.7 Hz, H-2', $6^{\prime}$ ), 7.75 (br. s, $1 \mathrm{H}, \mathrm{H}-\beta), 8.19$ (dd, $1 \mathrm{H}, J=7.8 \mathrm{~Hz}$ and $1.5 \mathrm{~Hz}, \mathrm{H}-5)$.

4.2.11. Compound 3b. IR $\left(\mathrm{KBr}, \mathrm{cm}^{-1}\right): 1673 \mathrm{~cm}^{-1}(\mathrm{C}=\mathrm{O}) ;{ }^{1} \mathrm{H}$ $\mathrm{NMR}\left(300 \mathrm{MHz}, \mathrm{CDCl}_{3}, \delta / \mathrm{ppm}\right): 3.96$ (br. s, $2 \mathrm{H},-\mathrm{CH}_{2}-$ ), 7.21 (d, 2H, $J=8.4 \mathrm{~Hz}$ ), 7.29 (d, 2H, $J=8.4 \mathrm{~Hz}$ ), 7.43 (br. s, $1 \mathrm{H}, \mathrm{H}-2), 7.50-7.62(\mathrm{~m}, 3 \mathrm{H}, \mathrm{Ar}-\mathrm{H}), 8.57$ (br. d, $1 \mathrm{H}, \mathrm{J}=$ $7.8 \mathrm{~Hz}$ ).

4.2.12. Compound 5d. IR $\left(\mathrm{KBr}, \mathrm{cm}^{-1}\right): 1674 \mathrm{~cm}^{-1}(\mathrm{C}=\mathrm{O}) ;{ }^{1} \mathrm{H}$ NMR (300 MHz, $\left.\mathrm{CDCl}_{3}, \delta / \mathrm{ppm}\right): 2.91$ (s, $4 \mathrm{H}, \mathrm{H}_{2}-3$ and $\mathrm{H}_{2}-$ 4), 6.90-7.00 (m, $4 \mathrm{H}, 2 \times \mathrm{H}-\gamma$ and $2 \times \mathrm{H}-\delta), 7.24-7.52(\mathrm{~m}$, $12 \mathrm{H}, \mathrm{Ar}-\mathrm{H}$ and $2 \times \mathrm{H}-\beta)$.

4.2.13. Compound 5j. IR $\left(\mathrm{KBr}, \mathrm{cm}^{-1}\right): 1658 \mathrm{~cm}^{-1}(\mathrm{C}=\mathrm{O}) ;{ }^{1} \mathrm{H}$ NMR (300 MHz, $\left.\mathrm{CDCl}_{3}, \delta / p p m\right): 1.83$ (br. s, $4 \mathrm{H}, \mathrm{H}_{2}-4$ and $\mathrm{H}_{2}-5$ ), 2.62 (br. s, $4 \mathrm{H}, \mathrm{H}_{2}-3$ and $\mathrm{H}_{2}-6$ ), 6.88-7.09 (m, 5H, Ar-H and olefinic $\mathrm{H}), 7.26-7.38$ ( $\mathrm{m}, 7 \mathrm{H}, \mathrm{Ar}-\mathrm{H}$ and olefinic $\mathrm{H}$ ), 7.48 (br. d, $4 \mathrm{H}, J=7.2 \mathrm{~Hz}, 2 \times \mathrm{H}-2^{\prime}, 6^{\prime}$ ); ${ }^{13} \mathrm{C} \mathrm{NMR}$ $(75 \mathrm{MHz}) \delta: 27.15,27.80,123.27,126.95,128.55,128.70$, 135.27, 136.78, 139.40, 140.93, 194.00. FABMS: $m / z 341.4$ $(\mathrm{M}+\mathrm{H})^{+}$; Anal. Calcd for $\mathrm{C}_{25} \mathrm{H}_{24} \mathrm{O}$ (340.18): C, 88.20; H, 7.11\%. Found C, 87.91; H 6.98\%. ${ }^{1} \mathrm{H}$ and ${ }^{13} \mathrm{C}$ NMR and mass spectral data of some selected compounds are supplied in a separate file. See Supplementary Material available online at doi: 10.1155/2012/456097 online.

\section{Acknowledgments}

The financial assistance from the UGC-CAS and DSTPURSE programs, Department of Chemistry, is gratefully acknowledged. The authors also acknowledge the DSTFIST program to the Department of Chemistry, Jadavpur University, for providing the NMR spectral data. One of them (R. Mondal) is thankful to the UGC, New Delhi, for the award of a research fellowship.

\section{References}

[1] A. Lévai, "Synthesis of exocyclic $\alpha, \beta$-unsaturated ketones," Arkivoc, vol. 2004, no. 7, pp. 15-33, 2004.

[2] A. Lévai, "Synthesis of heterocyclic compounds by the reactions of exocyclic $\alpha, \beta$-unsaturated ketones," Journal of Heterocyclic Chemistry, vol. 41, no. 3, pp. 299-310, 2004.

[3] A. Lévai, K. E. Kövér, and J. Jekõ, "Fused heterocycles 11. Synthesis of tricyclic fused pyrazolines by the reaction of 3arylidenechromanones and 3-arylidene-1-thiochromanones with hydrazine," Arkivoc, vol. 2007, no. 8, pp. 26-39, 2007.

[4] A. Lévai and J. Jeko", "Oxazepines and thiazepines 46. Synthesis of tetracyclic 1, 5 benzothiazepines by the reaction of $\alpha, \beta, \gamma, \delta$ unsaturated ketones with 2-aminothiophenol," Arkivoc, no. 17, pp. 234-240, 2008.

[5] J. B. Harborne, The Flavonoids: Advances in Research Since 1986, Chapman \& Hall, London, UK, 1994.

[6] W. Heller and C. Tamm, Fortschritte der Chemie Organischer Naturstoffe, vol. 40, Wiley-VCH Verlag GmbH \& Co. KGaA, Weinheim, Germany, 1988.
[7] T. Shankar, R. Gandhidasan, and S. Venkataraman, "Synthesis and characterization and antiinflamamatory and antibacterial evaluation of 3-arylidene-7-methoxychroman-4-ones," Indian Journal of Chemistry B, vol. 50, pp. 1202-1207, 2011.

[8] T. P. Robinson, T. Ehlers, R. B. Hubbard et al., "Design, synthesis, and biological evaluation of angiogenesis inhibitors: aromatic enone and dienone analogues of curcumin," Bioorganic and Medicinal Chemistry Letters, vol. 13, no. 1, pp. 115117, 2003.

[9] T. P. Robinson, R. B. Hubbard, T. J. Ehlers, J. L. Arbiser, D. J. Goldsmith, and J. P. Bowen, "Synthesis and biological evaluation of aromatic enones related to curcumin," Bioorganic and Medicinal Chemistry, vol. 13, no. 12, pp. 4007-4013, 2005.

[10] A. T. Dinkova-Kostova, C. Abeygunawardana, and P. Talalay, "Chemoprotective properties of phenylpropenoids, bis(benzylidene)cycloalkanones, and related Michael reaction acceptors: correlation of potencies as phase 2 enzyme inducers and radical scavengers," Journal of Medicinal Chemistry, vol. 41, no. 26, pp. 5287-5296, 1998.

[11] D. Cheng, S. Valente, S. Castellano et al., "Novel 3,5-bis (bromohydroxybenzylidene)piperidin-4-ones as coactivatorassociated arginine methyltransferase 1 inhibitors: enzyme selectivity and cellular activity," Journal of Medicinal Chemistry, vol. 54, no. 13, pp. 4928-4932, 2011.

[12] J. R. Dimmock, M. P. Padmanilayam, G. A. Zello et al., "Cytotoxic analogues of 2,6-bis(arylidene)cyclohexanones," European Journal of Medicinal Chemistry, vol. 38, no. 2, pp. 169-177, 2003.

[13] A. Modzelewska, C. Pettit, G. Achanta, N. E. Davidson, P. Huang, and S. R. Khan, "Anticancer activities of novel chalcone and bis-chalcone derivatives," Bioorganic and Medicinal Chemistry, vol. 14, no. 10, pp. 3491-3495, 2006.

[14] C. Piantadosi, I. H. Hall, J. L. Irvine, and G. L. Carlson, "Cycloalkanones. 2. Synthesis and biological activity of $\alpha, \alpha^{\prime}$ dibenzylcycloalkanones," Journal of Medicinal Chemistry, vol. 16, no. 7, pp. 770-775, 1973.

[15] N. Singh, J. Pandey, A. Yadav et al., "A facile synthesis of $\alpha, \alpha^{\prime}$ (EE)-bis(benzylidene)-cycloalkanones and their antitubercular evaluations," European Journal of Medicinal Chemistry, vol. 44, no. 4, pp. 1705-1709, 2009.

[16] C. T. Yen, K. Nakagawa-Goto, T. L. Hwang et al., "Antitumor agents. 271: total synthesis and evaluation of brazilein and analogs as anti-inflammatory and cytotoxic agents," Bioorganic and Medicinal Chemistry Letters, vol. 20, no. 3, pp. 1037$1039,2010$.

[17] Y. Wan, X. M. Chen, L. L. Pang et al., "Synthesis and fluorescence properties of $\alpha, \alpha^{\prime}$-bis(substituted- benzylidene) cycloalkanones catalyzed by 1-methyl-3(2-(sulfooxy)ethyl)$1 \mathrm{H}$-imidazol-3-ium chloride," Synthetic Communications, vol. 40, no. 15, pp. 2320-2328, 2010.

[18] Z. Karimi-Jaberi and B. Pooladian, "A facile synthesis of $\alpha, \alpha^{\prime}$ bis(substituted benzylidene) cycloalkanones catalyzed by $p$ TSA under solvent-free conditions," Green Chemistry Letters and Reviews, vol. 5, no. 2, pp. 187-193, 2012.

[19] G. Sabitha, G. S. K. K. Reddy, K. B. Reddy, and J. S. Yadav, "Iodotrimethylsilane-mediated cross-aldol condensation: a facile synthesis of $\alpha, \alpha^{\prime}$-bis(substituted benzylidene)cycloalkanones," Synthesis, no. 2, pp. 263-266, 2004.

[20] Y. Zhu and Y. Pan, "A new lewis acid system palladium/TMSCl for catalytic aldol condensation of aldehydes with ketones," Chemistry Letters, vol. 33, no. 6, pp. 668-669, 2004.

[21] Z. G. Hu, J. Liu, P. L. Zeng, and Z. B. Dong, "Synthesis of $\alpha, \alpha^{\prime}$-bis(substituted benzylidene)ketones catalysed by 
a $\mathrm{SOCl}_{2} / \mathrm{EtOH}$ reagent," Journal of Chemical Research, no. 1, pp. 55-56, 2004.

[22] L. Wang, J. Sheng, H. Tian, J. Han, Z. Fan, and C. Qian, "A convenient synthesis of $\alpha, \alpha^{\prime}$-bis(substituted benzylidene)cycloalkanones catalyzed by $\mathrm{Yb}(\mathrm{OTf})_{3}$ under solventfree conditions," Synthesis, no. 18, pp. 3060-3064, 2004.

[23] Y. Q. Cao, Z. Dai, R. Zhang, and B. H. Chen, "Aldol condensations catalyzed by PEG400 and anhydrous $\mathrm{K}_{2} \mathrm{CO}_{3}$ without solvent," Synthetic Communications, vol. 35, no. 8, pp. 1045-1049, 2005.

[24] J. Li, W. Su, and N. Li, "Copper triflate-catalyzed crossaldol condensation: a facile synthesis of $\alpha, \alpha$ '-bis(substituted benzylidene) cycloalkanones," Synthetic Communications, vol. 35, no. 23, pp. 3037-3043, 2005.

[25] B. Das, P. Thirupathi, I. Mahender, and K. R. Reddy, "Convenient and facile cross-aldol condensation catalyzed by molecular iodine: an efficient synthesis of $\alpha, \alpha^{\prime}$-bis(substitutedbenzylidene) cycloalkanones," Journal of Molecular Catalysis A, vol. 247, no. 1-2, pp. 182-185, 2006.

[26] S. Bhagat, R. Sharma, and A. K. Chakraborti, "Dualactivation protocol for tandem cross-aldol condensation: an easy and highly efficient synthesis of $\alpha, \alpha^{\prime}$-bis(aryl/alkylmethylidene)ketones," Journal of Molecular Catalysis A, vol. 260, no. 1-2, pp. 235-240, 2006.

[27] U. Grošelj, D. Bevk, R. Jakše, A. Meden, B. Stanovnik, and J. Svete, "Stereoselective additions to the exocyclic $\mathrm{C}=\mathrm{C}$ bond of some $\alpha$-alkylidene-(+)-camphor derivatives," Tetrahedron Asymmetry, vol. 17, no. 8, pp. 1217-1237, 2006.

[28] M. A. Bigdeli, G. H. Mahdavinia, S. Jafari, and H. Hazarkhani, "Wet 2,4,6-trichloro[1,3,5] triazine (TCT) an efficient catalyst for synthesis of $\alpha, \alpha^{\prime}$-bis(substituted-benzylidene) cycloalkanones under solvent-free conditions," Catalysis Communications, vol. 8, no. 12, pp. 2229-2231, 2007.

[29] L. Q. Kang, G. H. Song, J. Y. Wang, and B. G. Wei, "Synthesis of $\beta, \beta^{\prime}$-bis(substituted benzylidene) cycloalkanones catalyzed by amino-functionalized ionic liquid," Journal of the Chinese Chemical Society, vol. 55, no. 5, pp. 1125-1128, 2008.

[30] J. J. Shrikhande, M. B. Gawande, and R. V. Jayaram, "Crossaldol and Knoevenagel condensation reactions in aqueous micellar media," Catalysis Communications, vol. 9, no. 6, pp. 1010-1016, 2008.

[31] L. T. An, J. P. Zou, and L. L. Zhang, "Polymer-supported sulphonic acid catalyzed cross-aldol condensation: an expeditious synthesis of $\alpha, \alpha^{\prime}$-bis(substituted benzylidene) cycloalkanones," Catalysis Communications, vol. 9, no. 3, pp. 349-354, 2008.

[32] H. Hazarkhani, P. Kumar, K. S. Kondiram, and I. M. S. Gadwal, "Highly selective claisen-schmidt condensation catalyzed by silica chloride under solvent-free reaction conditions," Synthetic Communications, vol. 40, no. 19, pp. 2887-2896, 2010.

[33] A. Hasaninejad, A. Zare, L. Balooty, H. Mehregan, and M. Shekouhy, "Solvent-free, cross-aldol condensation reaction using silica-supported, phosphorus-containing reagents leading to $\alpha, \alpha^{\prime}$-bis(arylidene) cycloalkanones," Synthetic Communications, vol. 40, no. 23, pp. 3488-3495, 2010.

[34] A. Habibi, E. Sheikhhosseini, M. Bigdeli, S. Balalaie, and E. Farrokhi, "Solvent free synthesis of $\alpha, \alpha$ '-bis(substitutedbenzylidene)cycloalkanones using covalently anchored sulfonic acid on silica gel $\left(\mathrm{SiO}_{2}-\mathrm{R}-\mathrm{SO}_{3} \mathrm{H}\right)$ as an efficient and reusable heterogeneous catalyst," International Journal of Organic Chemistry, vol. 1, pp. 143-147, 2011.

[35] S. Kumar and J. K. Makrandi, "A facile solvent free synthesis of 3-arylidenechroman-4-ones using grinding technique," EJournal of Chemistry, vol. 9, no. 3, pp. 1251-1256, 2012.
[36] A. F. M. M. Rahman, R. Ali, Y. Jahng, and A. A. Kadi, "A facile solvent free Claisen-Schmidt reaction: synthesis of $\alpha, \alpha^{\prime}$-bis-(substituted-benzylidene)cycloalkanones and $\alpha, \alpha^{\prime}$ bis-(substituted-alkylidene)cycloalkanones," Molecules, vol. 17, no. 1, pp. 571-583, 2012.

[37] L. Wu, H. Guo, X. Wang, and R. Wu, "Synthesis and structure characterization of novel 3-substituted-(thio)chroman-4-one derivatives," Chinese Journal of Organic Chemistry, vol. 32, no. 3, pp. 608-611, 2012.

[38] S. Bhagat, R. Sharma, D. M. Sawant, L. Sharma, and A. K. Chakraborti, "LiOH $\cdot \mathrm{H}_{2} \mathrm{O}$ as a novel dual activation catalyst for highly efficient and easy synthesis of 1,3-diaryl2-propenones by Claisen-Schmidt condensation under mild conditions," Journal of Molecular Catalysis A, vol. 244, no. 12, pp. 20-24, 2006.

[39] Y. K. Srivastava, "Ecofriendly microwave massisted synthesis of some chalcones," Rasayan Journal of Chemistry, vol. 1, pp. 884-886, 2008.

[40] S. D. Kumar and J. S. Sandhu, "An efficient green protocol for the synthesis of chalcones by a Claisen-schmidt reaction using bismuth(III)chloride as a catalyst under solvent-free condition," Green Chemistry Letters and Reviews, vol. 3, no. 4, pp. 283-286, 2010.

[41] V. Tiwari, P. Ali, and J. Meshram, "Microwave assisted synthesis of 3-(2-chloroquiolin-3-yl)-1-substituted phenyl prop2-en-1-ones using $\mathrm{K}_{2} \mathrm{CO}_{3}$ as a mild, cheap and inexpensive catalyst," International Journal of ChemTech Research, vol. 2, no. 2, pp. 1031-1035, 2010.

[42] M. R. Jayapal and N. Y. Sreedhar, "Anhydrous $\mathrm{K}_{2} \mathrm{CO}_{3}$ as catalyst for the synthesis of chalcones under microwave irradiation," Journal of Pharmaceutical Sciences and Research, vol. 2, no. 10, pp. 644-647, 2010.

[43] D. Kakati and J. C. Sarma, "Microwave assisted solvent free synthesis of 1,3-diphenylpropenones," Chemistry Central Journal, vol. 5, no. 1, article 8, 2011.

[44] A. K. Chakraborti and G. Kaur, "One-pot synthesis of nitriles from aldehydes under microwave irradiation: influence of the medium and mode of microwave irradiation on product formation," Tetrahedron, vol. 55, no. 46, pp. 13265-13268, 1999.

[45] A. K. Chakraborti, C. Selvam, G. Kaur, and S. Bhagat, "An efficient synthesis of benzothiazoles by direct condensation of carboxylic acids with 2-aminothiophenol under microwave irradiation," Synlett, no. 5, pp. 851-855, 2004.

[46] R. Kumar, C. Selvam, G. Kaur, and A. K. Chakraborti, "Microwave-assisted direct synthesis of 2-substituted benzoxazoles from carboxylic acids under catalyst and solvent-free conditions," Synlett, no. 9, pp. 1401-1404, 2005.

[47] H. F. Motiwala, R. Kumar, and A. K. Chakraborti, "Microwave-accelerated solvent- and catalyst-free synthesis of 4-aminoaryl/alkyl-7-chloroquinolines and 2-aminoaryl/ alkylbenzothiazoles," Australian Journal of Chemistry, vol. 60, no. 5, pp. 369-374, 2007.

[48] S. Caddick, "Microwave assisted organic reactions," Tetrahedron, vol. 51, pp. 10403-10432, 1995.

[49] A. Loupy, A. Petit, J. Hamelin, F. Texier-Boullet, P. Jacquault, and D. Mathé, "New solvent-free organic synthesis using focused microwaves," Synthesis, no. 9, pp. 1213-1234, 1998.

[50] P. Lidström, J. Tierney, B. Wathey, and J. Westman, "Microwave assisted organic synthesis-a review," Tetrahedron, vol. 57, no. 45, pp. 9225-9283, 2001.

[51] B. Matthias, U. Müller, B. Ondruschka, A. Tied, and W. Lautenschläger, "Microwave-assisted chemical reactions," 
Chemical Engineering and Technology, vol. 26, no. 12, pp. 1207-1216, 2003.

[52] B. L. Hayes, "Recent advances in microwave-assisted synthesis," Aldrichimica Acta, vol. 39, pp. 66-76, 2004.

[53] H. M. Hügel, "Microwave multicomponent synthesis," Molecules, vol. 14, no. 12, pp. 4936-4972, 2009.

[54] A. K. Nagariya, A. K. Meena, Kiran et al., "Microwave assisted organic reaction as new tool in organic synthesis," Journal of Pharmacy Research, vol. 3, pp. 575-580, 2010.

[55] A. Loupy, Microwaves in Organic Synthesis, Wiley-VCH, Weinheim, Germany, 2002.

[56] D. Bogdal, Microwave-Assisted Organic Synthesis: One Hundred Reaction Procedures, Elsevier, Amsterdam, The Netherlands, 2005.

[57] E. van der Eycken, C. O. Kappe, and F. Almqvist, MicrowaveAssisted Synthesis of Heterocycles, Springer, Berlin, Germany, 2006.

[58] R. Mondal, A. Das Gupta, and A. K. Mallik, "Synthesis of flavanones by use of anhydrous potassium carbonate as an inexpensive, safe, and efficient basic catalyst," Tetrahedron Letters, vol. 52, no. 39, pp. 5020-5024, 2011.

[59] T. K. Mandal, R. Pal, R. Mondal, and A. K. Mallik, "Facile condensation of aromatic aldehydes with chroman-4-ones and 1-thiochroman-4-ones catalysed by amberlyst-15 under microwave irradiation condition," E-Journal of Chemistry, vol. 8, no. 2, pp. 863-869, 2011.

[60] T. Al Nakib, V. Bezjak, M. J. Meegan, and R. Chandy, "Synthesis and antifungal activity of some 3-benzylidenechroman-4ones, 3-benzylidenethiochroman-4-ones and 2-benzylidene1-tetralones," European Journal of Medicinal Chemistry, vol. 25, no. 5, pp. 455-462, 1990.

[61] R. Huisgen and W. Rapp, "Mittlere Ringe, I. Mitteil.: 1. 2benzo-cycloocten-(1)-on-(3)," Chemische Berichte, vol. 85, pp. 826-835, 1952.

[62] D. N. Kevill, E. D. Weiler, and N. H. Cromwell, "Elimination reactions of $\alpha$-halogenated ketones. XIII.1 substituent effects upon the kinetics and mechanism of halide ion promoted dehydrobromination of derivatives of 2-benzyl-2-bromo-1indanone in acetonitrile," Journal of the American Chemical Society, vol. 88, no. 19, pp. 4489-4494, 1966.

[63] T. Patonay, Z. Dinya, A. Lévai, and D. Molnár, "Reactivity of $\alpha$ arylidene benzoheteracyclanone dibromides toward azide ion: an effective approach to 3-( $\alpha$-substituted-benzyl)chromones and -1-thiochromones," Tetrahedron, vol. 57, no. 14, pp. 28952907, 2001.

[64] R. Pal, T. K. Mandal, C. Guha, and A. K. Mallik, "Amberlyst15 catalysed microwave assisted cross-aldol condensation between ketones and aldehydes under solvent free condition," Journal of the Indian Chemical Society, vol. 88, pp. 711-717, 2011.

[65] N. T. Leonard, L. A. Miller, and J. W. Berry, "The synthesis of 2,7-disubstituted tropones via aromatization," Journal of the American Chemical Society, vol. 79, no. 6, pp. 1482-1485, 1957. 


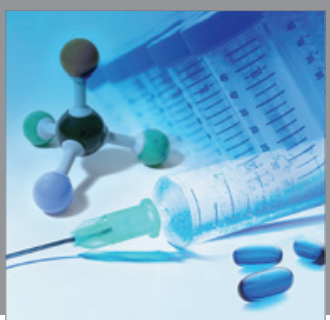

International Journal of

Medicinal Chemistry

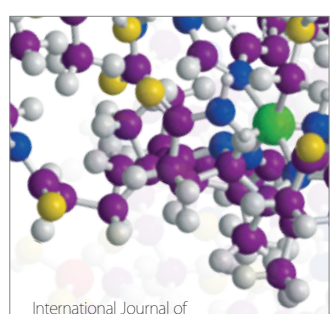

Carbohydrate Chemistry

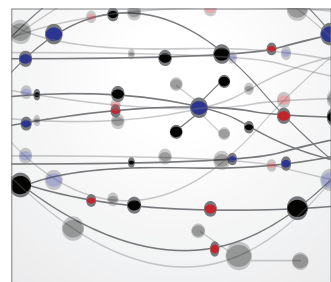

The Scientific World Journal
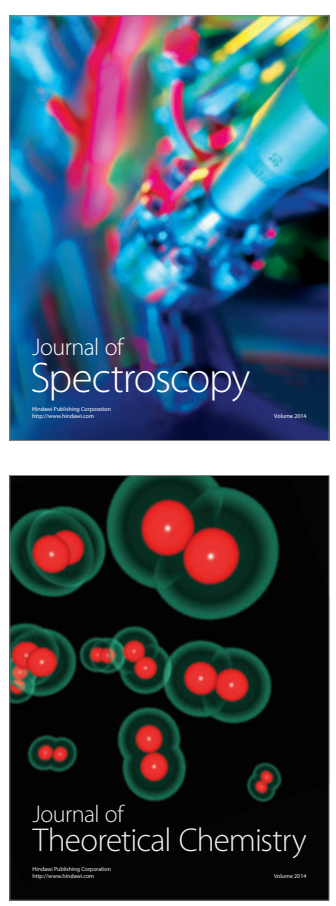
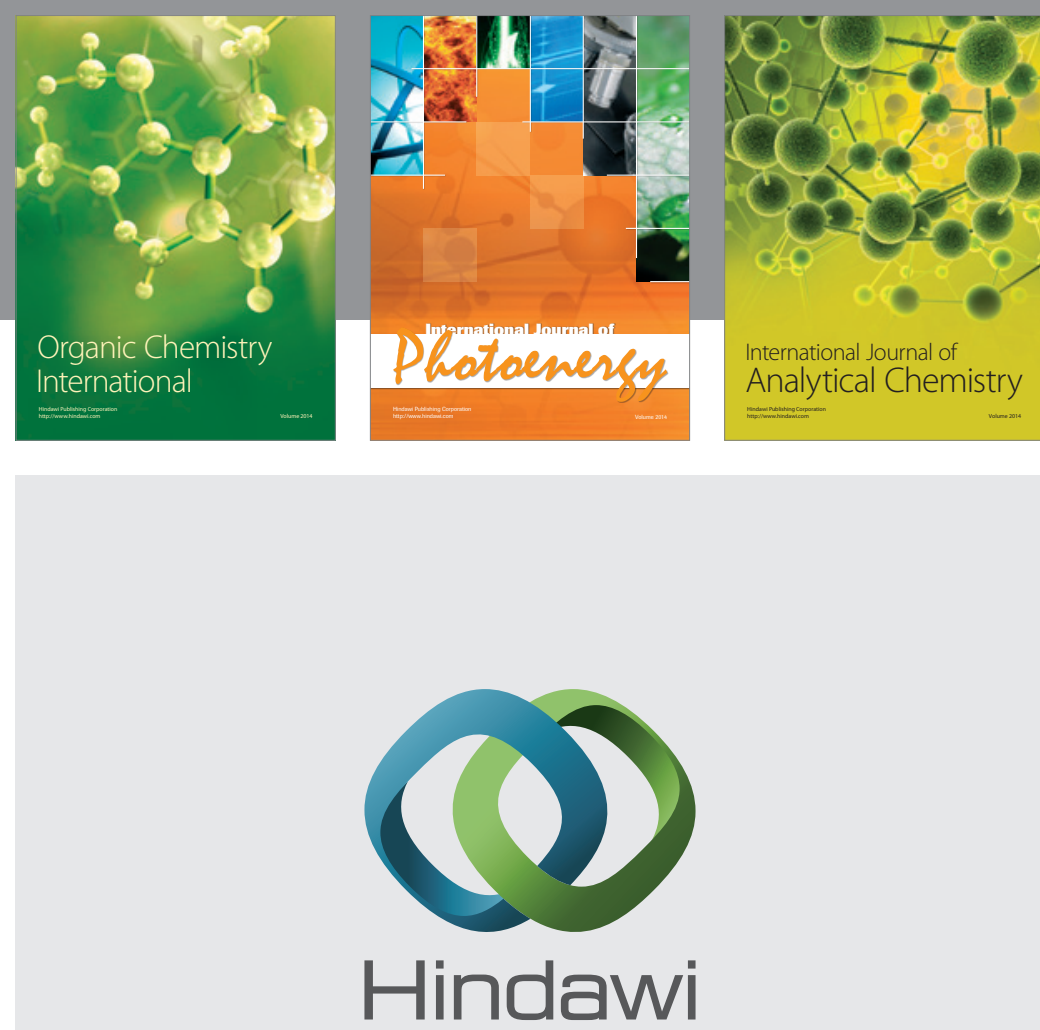

Submit your manuscripts at

http://www.hindawi.com
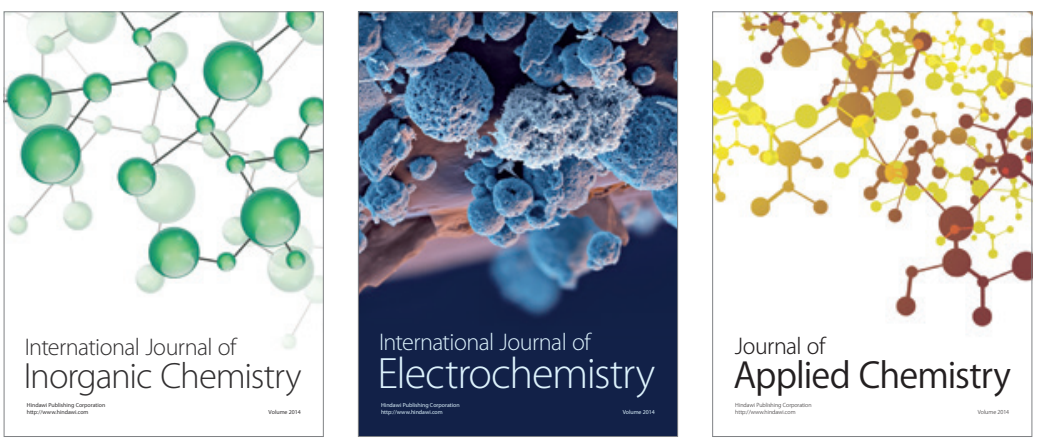

Journal of

Applied Chemistry
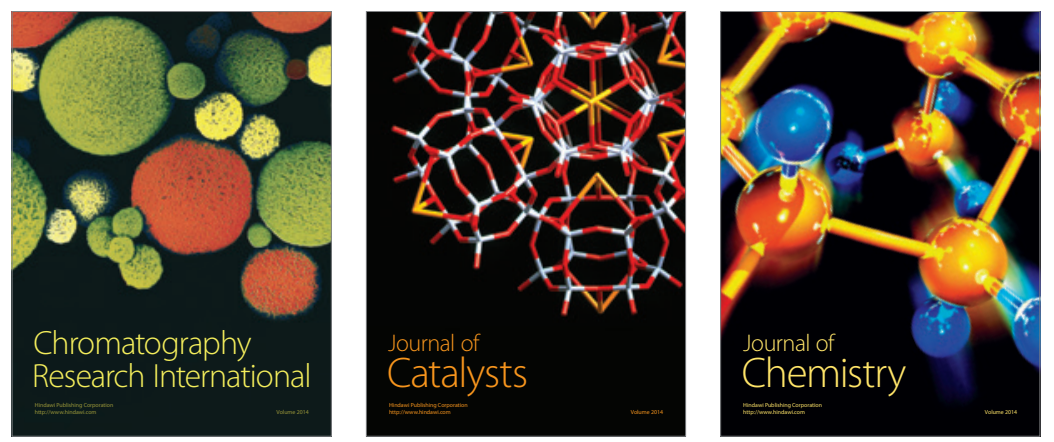
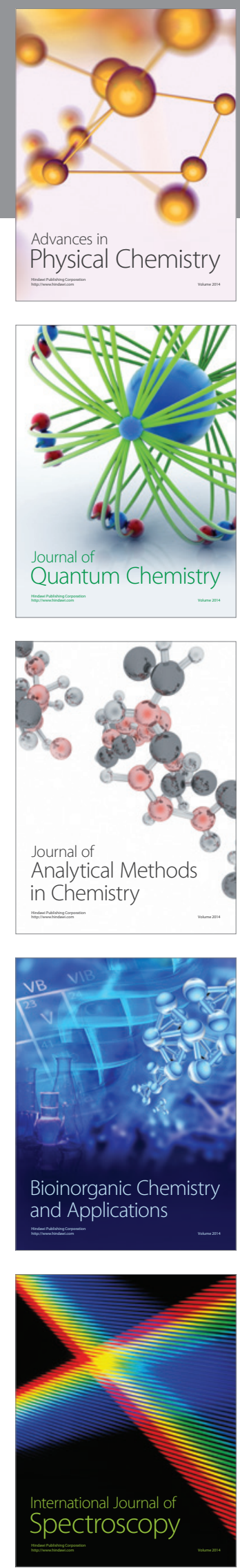